Bull. Austral. Math. Soc.

VOL. $72(2005)$ [461-470]

\title{
APPROXIMATE SOLUTIONS FOR THE COUETTE VISCOMETRY EQUATION
}

\author{
F.R. DE HoOg AND R.S. ANDERSSEN
}

\begin{abstract}
The recovery of flow curves for non-Newtonian fluids from Couette rheometry measurements involves the solution of a quite simple first kind Volterra integral equation with a discontinuous kernel for which the solution, as a summation of an infinite series, has been known since 1953. Various methods, including an Euler-Maclaurin sum formula, have been proposed for the estimation of the value of the summation. They all involve the numerical differentiation of the observational data. In this paper, the properties of Bernoulli polynomials, in conjunctions with the special structure of the integral equation, are exploited to derive a parametric family of representations for its solution. They yield formulas similar to, but more general than, the previously published Euler-Maclaurin sum formula representations. The parameterisation is then utilised to derive two new classes of approximations. The first yields a family of finite difference approximations, which avoids the direct numerical differentiation of the observational data, while the second generates a framework for the construction of improved power law approximations.
\end{abstract}

\section{INTRODUCtion - THE COUETTE RHEOMETER EQUation}

The growing sophistication of material science is generating a demand for improved or new instrumentation for determining the rheological characteristics of non-Newtonian fluids [3]. In fact, improved instrumentation has stimulated, from a numerical analysis perspective, a review of the traditional and historic algorithms for the recovery of flow curves (see [2]). This includes algorithms specifically developed for Couette rheometer data, because of the popularity of this device in industrial applications $([18,14])$.

A Couette (concentric cylinder) rheometer $([5,17])$ consists of the inner cylinder (the bob) of length $L$ and radius $R_{b}$ and the outer cylinder (the cup) of radius $R_{c}$, where the cup is rotated at an angular velocity $\Omega$ while a torque $M$ is applied to the bob to hold it stationary. For an ideal Couette flow, equilibrium requires that the shear stress $\sigma(r)$ exerted on the fluid between the two cylinders as a radius $r, R_{b} \leqslant r \leqslant R_{c}$, takes the form

$$
\sigma(r)=\frac{M}{2 \pi r^{2} L}
$$

Received 2nd Ausugt, 2005

Copyright Clearance Centre, Inc. Serial-fee code: 0004-9727/05 \$A2.00+0.00. 
For such flows, the constitutive relationship can be formulated to take the form

$$
\dot{\gamma}(\sigma)=\sigma f(\sigma),
$$

which states that, for a perfect liquid in Couette flow, the rate of shearing $\dot{\gamma}$ is only a function of the form $\sigma f(\sigma)$ of the local shear stress $\sigma$. Here, following [15], $f(\sigma)$ denotes the fluidity which is the reciprocal of the viscosity.

As first derived by [15] and rederived subsequently by various authors, the Couette rheometer equation takes the following form

$$
2 \Omega(\sigma)=\int_{s^{2} \sigma}^{\sigma} f(\eta) d \eta, \quad \sigma=\frac{M}{2 \pi R_{b}^{2} L}, \quad s=\frac{R_{b}}{R_{c}} .
$$

It corresponds to a first kind integral equation with the discontinuous kernel

$$
H\left(\eta-s^{2} \sigma\right)-H(\eta-\sigma),
$$

where $H(\cdot)$ denotes the Heaviside Unit Step function which takes the value 1 for positive arguments and is zero otherwise.

Differentiation of equation (1), with respect to $\sigma$, yields the result first derived by [15]

$$
2 \frac{d \Omega}{d \sigma}=f(\sigma)-s^{2} f\left(s^{2} \sigma\right)
$$

In [15], Mooney then applied Taylor series expansions to derive his numerical algorithm. Krieger and Maron [13] proposed alternative methods based on this expression. The key step, implemented independently by Krieger and Elrod [12] and Pawlowski [16], is to iterate this result. It yields the infinite series solution

$$
f(\sigma)=2 \sum_{j \geqslant 0} s^{2 j} \Omega^{\prime}\left(s^{2 j} \sigma\right), \quad \Omega^{\prime}=\frac{d \Omega}{d \sigma} .
$$

An alternative derivation has been given in [6]. It was Krieger and Elrod [12] who made the fundamental observation that the Euler-Maclaurin sum formula could be applied to the sum in this expression, though in a form different from that presented here. For algebraic reasons, related to the manipulations to be performed in subsequent section, it is convenient to invoke equation (1) to rewrite equation (2) in the form

$$
2 \Omega(\sigma)=\int_{s^{2} \sigma}^{\sigma} \frac{\dot{\gamma}(\eta)}{\eta} d \eta
$$

and then introduce the substitution $\eta=\exp (\beta)$ to obtain

$$
2 \Omega(\sigma)=\int_{\ln (\sigma)-\varepsilon}^{\ln (\sigma)} \dot{\gamma}(\exp (\beta)) d \beta, \quad \varepsilon=-2 \ln (s)=2 \ln \left(\frac{R_{c}}{R_{b}}\right)
$$


or, equivalently, as

$$
\omega(z)=\int_{z-\varepsilon}^{z} g(\beta) d \beta
$$

where

$$
\omega(z)=2 \Omega(\exp (z)), \quad g(z)=\dot{\gamma}(\exp (z))
$$

\section{The Bernoulli polynomial solution}

It is well-known that the generating function of the Bernoulli polynomials $B_{k}(x)$ takes the form $([\mathbf{1}])$

$$
\frac{t \exp (x t)}{\exp (t)-1}=\sum_{k \geqslant 0} B_{k}(x) \frac{t^{k}}{k !}, \quad|t|<2 \pi
$$

where the $B_{k}(\cdot)$ denotes the Bernoulli polynomial of degree $k$. The Bernoulli numbers $B_{k}$ are given by

$$
B_{k}=B_{k}(0), \quad B_{0}=1, \quad B_{1}=-\frac{1}{2}, \quad B_{2}=\frac{1}{6}, \quad B_{4}=-\frac{1}{30}, \cdots
$$

with

$$
B_{2 k+1}=0, \quad k=1,2, \cdots
$$

The relationships

$$
B_{k}(1)=(-1)^{k} B_{k}, \quad \text { and } \quad B_{k}(1 / 2)=-\left(1-2^{1-k}\right) B_{k},
$$

allow values of $B_{k}(1)$ and $B_{k}(1 / 2)$ to be determined when required.

Using Taylor's theorem, the following formal equivalence, which is central to the subsequent deliberations, can be constructed

$$
g(x+\varepsilon)=\sum_{k \geqslant 0} \frac{\varepsilon^{k}}{k !} g^{(k)}(x)=\left\{\sum_{k \geqslant 0} \frac{\varepsilon^{k} D^{(k)}}{k !}\right\} g(x)=\exp (\varepsilon D) g(x) .
$$

Formally, because the actions of the operators $D$ and $\exp (-\varepsilon \alpha D)$ commute, and $D D^{-1}$ $=I$, the identity operator, it follows that

$$
\begin{aligned}
\varepsilon \exp (-\varepsilon \alpha D) & =\varepsilon D \exp (-\varepsilon \alpha D)(1-\exp (-\varepsilon D))^{-1}(1-\exp (-\varepsilon D)) D^{-1} \\
& =\varepsilon D \exp (\varepsilon(1-\alpha) D)(\exp (\varepsilon D)-1)^{-1}(1-\exp (-\varepsilon D)) D^{-1} \\
& =\left\{\sum_{k \geqslant 0} B_{k}(1-\alpha) \frac{(\varepsilon D)^{k}}{k !}\right\}(1-\exp (-\varepsilon D)) D^{-1} .
\end{aligned}
$$


Combining these two results then yields the following identity

$$
\begin{aligned}
\varepsilon g(x-\varepsilon \alpha) & =\{\varepsilon \exp (-\varepsilon \alpha D)\} g(x) \\
& =\left\{\sum_{k \geqslant 0} B_{k}(1-\alpha) \frac{(\varepsilon D)^{k}}{k !}\right\}(1-\exp (-\varepsilon D)) D^{-1} g(x) \\
& =\sum_{k \geqslant 0} B_{k}(1-\alpha) \frac{\varepsilon^{k} D^{k}}{k !} \int_{x-\varepsilon}^{x} g(\beta) d \beta .
\end{aligned}
$$

Substitution from equation (3) into this result thereby yields the following family of parameterised representations for the solution of equation (3)

$$
\begin{aligned}
\varepsilon g(x-\varepsilon \alpha) & =\sum_{k \geqslant 0} B_{k}(1-\alpha) \frac{\varepsilon^{k}}{k !} D^{k} \omega(x) \\
& =\omega(x)+\sum_{k \geqslant 1} B_{k}(1-\alpha) \frac{\varepsilon^{k}}{k !} \frac{d^{k} \omega(x)}{d x^{k}} .
\end{aligned}
$$

More details about, including a more rigorous derivation of, Euler-Maclaurin sum formulas and related mathematical results can be found in [8]. Through the judicious choice of the values of the parameter $\alpha$, one is able to derive new approximations for the solution of the Couette rheometer equation. Finite difference formulas are derived in Section 3, while a new correction procedure for the power law approximation of [9], along the lines investigated by Code and Raal [4] and Krieger [11], is derived in Section 4.

\section{THE FINITE DIFFERENCE APPROXIMATIONS}

Because it corresponds to a local analysis, the derivation below is quite different from that of Krieger and Elrod [12], which took as its starting point the full series solution. On setting $\alpha=0$ in equation (5) and invoking the appropriate values of the Bernoulli numbers $B_{k}$, one obtains the following counterpart of the Euler-Maclaurin sum formula solution proposed by Krieger and Elrod [12].

$$
\varepsilon g(x)=\omega(x)+\frac{\varepsilon}{2} \frac{d \omega(x)}{d x}+\frac{\varepsilon^{2}}{12} \frac{d^{2} \omega(x)}{d x^{2}}+\sum_{k \geqslant 2} B_{2 k} \frac{\varepsilon^{2 k}}{(2 k) !} \frac{d^{2 k} \omega(x)}{d x^{2 k}} .
$$

On setting $\alpha=1 / 2$ in equation (5), which corresponds to the mid-point form of the EulerMaclaurin sum formula, one obtains the following more accurate and simpler formula, because the first derivative term has been eliminated,

$$
\varepsilon g\left(x-\frac{\varepsilon}{2}\right) \approx \omega(x)-\frac{\varepsilon^{2}}{24} \frac{d^{2} \omega(x)}{d x^{2}}-\sum_{k \geqslant 2}\left(1-2^{1-2 k}\right) B_{2 k} \frac{\varepsilon^{2 k}}{(2 k) !} \frac{d^{2 k} \omega(x)}{d x^{2 k}}
$$


For the finite difference approximation of the second derivative of a function on an even-grid, Taylor's theorem yields

$$
\omega(x+\varepsilon)-2 \omega(x)+\omega(x-\varepsilon)=\varepsilon^{2} \frac{d^{2} \omega(x)}{d x^{2}}+2 \sum_{k \geqslant 2} \frac{\varepsilon^{2 k}}{(2 k) !} \frac{d^{2 k} \omega(x)}{d x^{2 k}} .
$$

Substitution of this result for the second derivative term on the right hand side of equation (6) then yields

$$
\begin{aligned}
\varepsilon g\left(x-\frac{\varepsilon}{2}\right)=-\frac{1}{24}\{\omega(x+\varepsilon)-26 \omega(x) & +\omega(x-\varepsilon)\} \frac{3}{640} \varepsilon^{4} \frac{d^{4} \omega(x)}{d x^{4}}+ \\
& +\sum_{k \geqslant 3}\left[\frac{1}{12}-\left(1-2^{1-2 k}\right) B_{2 k}\right] \frac{\varepsilon^{2 k}}{(2 k) !} \frac{d^{2 k} \omega(x)}{d x^{2 k}}
\end{aligned}
$$

Equations (6) and (7) can be rearranged to yield, respectively, the following two finite difference approximations for $\omega(x)$

$$
g(x) \approx \frac{1}{\varepsilon} \omega\left(x+\frac{1}{2} \varepsilon\right)
$$

and

$$
g(x) \approx-\frac{1}{24 \varepsilon}\left\{\omega\left(x+\frac{3}{2} \varepsilon\right)-26 \omega\left(x+\frac{1}{2} \varepsilon\right)+\omega\left(x-\frac{1}{2} \varepsilon\right)\right\} .
$$

In terms of the original variables, allowing for the fact that $\ln (s)$ will be negative, these two finite difference approximations become

$$
\dot{\gamma}(\sigma) \approx \frac{\Omega\left(\sigma s^{-1}\right)}{(-\ln (s))}
$$

and

$$
\dot{\gamma}(\sigma) \approx \frac{1}{(-24 \ln (s))}\left\{-\Omega\left(\sigma s^{-3}\right)+26 \Omega\left(\sigma s^{-1}\right)-\Omega(\sigma s)\right\}
$$

Proceeding in a similar manner, one can derive the following higher order approximation

$$
\dot{\gamma}(\sigma) \approx \frac{9 \Omega\left(\sigma s^{-5}\right)-116 \Omega\left(\sigma s^{-3}\right)+2134 \Omega\left(\sigma s^{-1}\right)-116 \Omega(\sigma s)+9 \Omega\left(\sigma s^{3}\right)}{(-1920 \ln (s))} .
$$

The major advantage of these finite difference approximations is that they can be applied directly without the need to perform an explicit numerical differentiation of the measured data. Clearly, the underlying improperly posedness, in terms of the implicit differentiation associated with the recovery of $\dot{\gamma}(\sigma)$-values from measured values of $\Omega$ (see [6]), is hidden in the oscillating positive and negative signs in the second two formulas. Equally importantly, these formulas define the grid on which the values of $\Omega$ can be measured so that they can be applied directly without the need to interpolate the measured values onto an appropriate grid. Nevertheless, it is more than likely that measurements will be performed with error on some other, possibly finer, grid generating the need to 
smooth the data before applying one of the finite difference formulas. In order to cope with only having a small number of data, Ancey [2] used the optimised Gasser-Müller kernel method $([10])$ to perform the smoothing before applying his wavelet-vaguelette procedure. In that paper, methods based on the wavelet-vaguelette decomposition of [7] are proposed and analysed for the inversion of Couette rheometry data.

\section{A MODIFIED POWER LAW APPROXIMATION}

As noted by Krieger [11] and Farrow, Lowe and Neale [9] were the first to propose and analyse a power law model of the form

$$
\dot{\gamma}(\sigma)=a \sigma^{\lambda}
$$

for the flow in a Couette rheometer from which it follows that

$$
\dot{\gamma}(\sigma)=\frac{2 \lambda \Omega(\sigma)}{1-s^{-2 \lambda}}, \quad \lambda=\sigma \frac{\Omega^{\prime}(\sigma)}{\Omega(\sigma)} .
$$

In [4], Code and Rall in terms of their concept of a power law factor, conceptualised the idea that, for general non-Newtonian fluids, the exponent $\lambda$ could be assumed to be a function of $\sigma$. By exploiting the properties of the Euler-Maclaurin sum formula solution in $[12,11]$, noted that the power law factor could be determined as a correction factor added to the [9] result. In terms of the notation of equation (3) and the power law approximation with $\lambda=\lambda(\sigma)$, it follows that

$$
g(x)=c \exp (\lambda(x) x)
$$

For $\lambda(x)=\bar{\lambda}=$ constant, it follows that

$$
\omega(x)=c \exp (\bar{\lambda} x) \frac{(1-\exp (\bar{\lambda} \varepsilon))}{\bar{\lambda}} .
$$

and, hence,

$$
\bar{\lambda}=\frac{\omega^{\prime}(x)}{\omega(x)}, \quad \omega^{\prime}(x)=\frac{d \omega(x)}{d x} .
$$

Furthermore, one obtains from equation (3) that

$$
\sum_{k \geqslant 0} \omega(x-k \varepsilon)=\int_{-\infty}^{x} g(\beta) d \beta
$$

and, hence,

$$
g(x)=\sum_{k \geqslant 0} \omega^{\prime}(x-k \varepsilon)=\bar{\lambda} \sum_{k \geqslant 0} \omega(x-k \varepsilon)=\bar{\lambda} \sum_{k \geqslant 0} \omega(x) \exp (-k \varepsilon)=\frac{\bar{\lambda} \omega(x)}{1-\exp (-\varepsilon)} .
$$


It is therefore natural to assume that $\lambda(\sigma)$ adjusts to the changing value of $\sigma$ such that

$$
\lambda(x)=\frac{\omega^{\prime}(x)}{\omega(x)}
$$

Using the Bernoulli polynomial solution of Section 2, these ideas can be formalised in the following manner. On rewriting equation (8) in the form

$$
\omega^{\prime}(x)=\lambda(x) \omega(x)
$$

differentiation, with respect to $x$, and the application of equation (8) to remove the derivative $\omega^{\prime}(x)$ yields

$$
\omega^{(2)}(x)=\lambda^{2}(x) \omega(x)+\lambda^{\prime}(x) \omega(x)
$$

and hence

$$
\omega^{(3)}(x)=\lambda^{3}(x) \omega(x)+3 \lambda(x) \lambda^{\prime}(x) \omega(x)+\cdots
$$

Continuing the process inductively yields

$$
\omega^{(k)}(x)=\lambda^{k}(x) \omega(x)+\frac{k(k-1)}{2}(\lambda(x))^{k-2} \lambda^{\prime}(x) \omega(x)+\cdots .
$$

Incorporation of these results into equation (5) gives

$$
\begin{aligned}
\varepsilon g(x-\varepsilon \alpha)= & \sum_{k \geqslant 0} B_{k}(1-\alpha) \frac{\varepsilon^{k}}{k !} D^{k} \omega(x) \\
=\omega(x) & \sum_{k \geqslant 0} B_{k}(1-\alpha) \frac{(\lambda(x) \varepsilon)^{k}}{k !} \\
& +\frac{1}{2} \varepsilon^{2} \omega(x) \lambda^{\prime}(x) \sum_{k \geqslant 2} B_{k}(1-\alpha) \frac{k(k-1)(\lambda(x) \varepsilon)^{k-2}}{k !}+\cdots \\
=\varepsilon \omega(x) & \frac{\lambda(x) \exp (-\alpha \lambda(x) \varepsilon)}{1-\exp (-\lambda(x) \varepsilon)} \\
& +\frac{1}{2} \omega(x) \frac{\lambda^{\prime}(x)}{\lambda^{2}(x)}(\lambda(x) \varepsilon)^{2} \sum_{k \geqslant 2} B_{k}(1-\alpha) \frac{k(k-1)(\lambda(x) \varepsilon)^{k-2}}{k !}+\cdots
\end{aligned}
$$

Rewriting the generating function (4) for the Bernoulli polynomials in the form

$$
\frac{t \exp (p t)}{\exp (t)-1}=\sum_{k \geqslant 0} B_{k}(p) \frac{t^{k}}{k !}, \quad p=1-\alpha, \quad|t|<2 \pi
$$


it follows that

$$
\begin{aligned}
& t^{2} \sum_{k \geqslant 2} B_{k}(p) \frac{k(k-1) t^{k-2}}{k !} \\
& \quad=t^{2} \frac{d^{2}}{d t^{2}}\left(\frac{t \exp (p t)}{\exp (t)-1}\right) \\
& \quad=t^{2}\left\{\frac{2 t \exp (t p+2 t)}{(\exp (t)-1)^{3}}+\frac{\exp (t p+t)}{(\exp (t)-1)^{2}}[-t p-t(1+p)-2]+\frac{\exp (t p)}{(\exp (t)-1)}\left[2 p+t p^{2}\right]\right\} \\
& \quad=\frac{t \exp (t p)}{(\exp (t)-1)}\left\{t\left[\frac{2 t \exp (2 t)}{(\exp (t)-1)^{2}}-\frac{(t+2 t p+2) \exp (t)}{(\exp (t)-1)}+p(2+t p)\right]\right\} .
\end{aligned}
$$

Combining this last result with equation (9), one obtains

$$
\begin{aligned}
\varepsilon g(x-\varepsilon \alpha)=\omega(x) \varepsilon \lambda(x) \frac{\exp (-\alpha \lambda(x) \varepsilon)}{1-\exp (-\lambda(x) \varepsilon)} \\
\quad+\frac{1}{2} \omega(x) \frac{\lambda^{\prime}(x)}{\lambda^{2}(x)}(\lambda(x) \varepsilon)^{2} \sum_{k \geqslant 2} B_{k}(1-\alpha) \frac{k(k-1)(\lambda(x) \varepsilon)^{k-2}}{k !}+\cdots \\
=\omega(x) \varepsilon \lambda(x) \frac{\exp (-\alpha \lambda(x) \varepsilon)}{1-\exp (-\lambda(x) \varepsilon)}\left\{1+\frac{\lambda^{\prime}(x)}{\lambda^{2}(x)} h(t(x))+\cdots\right\}
\end{aligned}
$$

where

$$
h(t)=\frac{t}{2}\left\{\frac{2 t \exp (2 t)}{(\exp (t)-1)^{2}}-\frac{(2+2 t p+t) \exp (t)}{(\exp (t)-1)}+p(2+t p)\right\}, \quad t(x)=\varepsilon \lambda(x), \quad p=1-\alpha .
$$

In order to exploit the parametric structure of equation (10) for the generation of more efficient approximations than those derived in [11], it is first necessary to derive an appropriate approximation for $h(t)$. It follows from its definition that

$$
\begin{aligned}
h(t) & =\frac{t}{2}\left\{\frac{2 t \exp (t)(\exp (t)-1+1)}{(\exp (t)-1)^{2}}-\frac{(2+2 t p+t) \exp (t)}{(\exp (t)-1)}+p(2+t p)\right\} \\
& =\frac{t}{2}\left\{\frac{2 t \exp (t)}{(\exp (t)-1)^{2}}-\frac{(2+2 t p-t)((\exp (t)-1)+1)}{(\exp (t)-1)}+p(2+t p)\right\} \\
& =\frac{t}{2}\left\{\frac{2 t((\exp (t)-1)+1)}{(\exp (t)-1)^{2}}-\frac{(2+2 t p-t)}{(\exp (t)-1)}+p(2+t p)-(2+2 t p-t)\right\} \\
& =\frac{t}{2}\left\{\frac{2 t}{(\exp (t)-1)^{2}}-\frac{(2+2 t p-3 t)}{(\exp (t)-1)}+t(1-p)^{2}-2(1-p)\right\} \\
& =\frac{t}{2}\left\{-\left[2 \frac{1-t}{(\exp (t)-1)}-\frac{t}{(\exp (t)-1)^{2}}\right]-\frac{(2 p-1) t}{(\exp (t)-1)}+t(1-p)^{2}-2(1-p)\right\} \\
& =\frac{t}{2}\left\{-2 \frac{d}{d t}\left(\frac{t}{(\exp (t)-1)}\right)-(2 p-1) \frac{t}{(\exp (t)-1)}+t(1-p)^{2}-2(1-p)\right\} \\
& =\frac{t}{2}\left\{-2 \frac{d}{d t}\left(\sum_{k \geqslant 0} B_{k} \frac{t^{k}}{k !}\right)-(2 p-1) \sum_{k \geqslant 0} B_{k} \frac{t^{k}}{k !}+t(1-p)^{2}-2(1-p)\right\}
\end{aligned}
$$




$$
\begin{aligned}
& =\frac{t}{2}\left\{-2 \sum_{k \geqslant 0} B_{k+1} \frac{t^{k}}{k !}-(2 p-1) \sum_{k \geqslant 0} B_{k} \frac{t^{k}}{k !}+t(1-p)^{2}-2(1-p)\right\} \\
& =\frac{t}{2}\left\{-2 B_{2} t-(2 p-1) B_{1} t+t(1-p)^{2}+O\left(t^{3}\right)\right\} \\
& =\frac{t^{2}}{2}\left\{-\frac{1}{3}+\frac{1}{2}(2 p-1)+(1-p)^{2}\right\}+O\left(t^{3}\right)=\frac{t^{2}}{12}\left\{6 p^{2}-6 p+1\right\}+O\left(t^{3}\right) .
\end{aligned}
$$

Substitution of this result into equation (10) yields

$$
\varepsilon g(x-\varepsilon \alpha)=\omega(x) \varepsilon \lambda(x) \frac{\exp (-\alpha \lambda(x) \varepsilon)}{1-\exp (-\lambda(x) \varepsilon)}\left\{1+\varepsilon \frac{\lambda^{\prime}(x)}{\lambda(x)}\left[\frac{t^{2}}{12}\left(6 p^{2}-6 p+1\right)+O\left(t^{3}\right)\right]+\cdots\right\},
$$

The construction of new approximations is thereby reduced to determining the value of $\alpha$ that simplify this last expression in one way or another. One obvious strategy is to choose $\alpha$ such that $6 p^{2}-6 p+1=0, p=1-\alpha$; namely, choose $\alpha^{*}=1 / 2-\sqrt{1 / 12}$, since it is closest to the power law result first derived by [9]. This leads naturally to the new approximation

$$
\dot{\gamma}(\sigma) \approx 2 \frac{\Omega\left(\sigma_{1}\right) \lambda\left(\sigma_{1}\right)}{\left(1-s^{2 \lambda\left(\sigma_{1}\right)}\right)} s^{2 \alpha \lambda\left(\sigma_{1}\right)}, \quad \sigma_{1}=\sigma s^{-2 \alpha}, \quad \lambda(\sigma)=\sigma \frac{\Omega^{\prime}(\sigma)}{\Omega(\sigma)} .
$$

Compared with the finite difference formulas derived in Section 3, this new approximation involves just a single differentiation of the measured values of $\Omega(\sigma)$.

\section{REFERENCES}

[1] M. Abramowitz and I.A. Stegun, Handbook of mathematical functions (Dover Publications, New York, 1965).

[2] C. Ancey, 'Solving the Couette inverse problem by using a wavelet-vaguelette decomposition', J. Rheol. 49 (2005), 441-460.

[3] J.C. Baudez and P. Coussot, 'Abrupt transition from viscoelastic solidlike to liquidlike behaviour in jammed materials', Phys. Review Let. 93 (2004), \#128302(4).

[4] R.K. Code and J.D. Raal, 'Rates of shear in coaxial cylinder viscometers', Rheol. Acta 12 (1973), 578-587.

[5] M. Couette, 'Études sur le frottement des liquides', Ann. Chim. Phys. 21 (1890), 433-510.

[6] F.R. de Hoog and R.S. Anderssen, 'Regularization of first kind integral equations with application to Couette viscometry', J. Integral Eqations Appl. (to appear).

[7] D. Donoho, 'Nonlinear solution of linear inverse problems by wavelet-vaguelette decomposition', Appl. Comput. Harmon. Anal. 2 (1995), 101-1126.

[8] D. Elliott, 'The Euler-Maclaurin formula revisited', J. Austral. Math. Soc. Ser. B 40 (1998), E27-E76.

[9] F.D. Farrow, G.M. Lowe and S.M. Neale, 'The flow of starch pastes flow at high and low rates of shear', J. Textile Inst. 19 (1928), T18-T31.

[10] J. Hart, Nonparametric smoothing and lack-of-fit tests (Springer, New York, 1999).

[11] I.M. Krieger, 'Shear rate in the Couette viscometer', Trans. Soc. Rheol. 12 (1968), 5-11. 
[12] I.M. Krieger and H. Elrod, 'Direct determination of the flow curves of non-Newtonian fluids. II. Shearing rate in the concentric cylinder viscometer', J. Appl. Phys. 24 (1953), 134-136.

[13] I.M. Krieger and S.H. Maron, 'Direct determination of the flow curves of non-Newtonian fluids,', J. Appl. Phys. 23 (1952), 147-149.

[14] Y.K. Leong and Y.L. Yeow, 'Obtaining the shear stress shear rate relationship and yield stress of liquid foods from Couette viscometry data', Rheol. Acta 42 (2003), 365-371.

[15] M. Mooney, 'Explicit formulas for slip and fluidity', J. Rheol. 2 (1931), 210-222.

[16] J. Pawlowski, 'Bestimmung des Reibungsgesetzes der nicht-Newtonschen Flüssigkeiten aus den Viskositätsmessungen mit Hilfe eines Rotationsviskosimeters', Kolloid Zeit. 10 (1953), 129-131.

[17] J.M. Piau, M. Bremond, J.M. Couette and M. Piau, 'Maurice Couette, one of the founders of rheology', Rheol. Acta 33 (1994), 357-368.

[18] C. Picart, J.M. Piau, H. Galliard and P. Carpenter, 'Human blood yield stress and its hematorit dependence', J. Rheol. 42 (1998), 1-12.

CSIRO Mathematical and Information Sciences

GPO Box 664

Canberra ACT 2601

Australia 